\section{Telarca Reversível Espontaneamente em Meninas Pré-Púberes Durante o Tratamento com Hormônio de Crescimento Recombinante Humano}

\section{RESUMO}

OBJETIVO: Descrição de 4 casos de desenvolvimento transitório de mamas em meninas pré-púberes tratadas com hormônio de crescimento recombinante humano (rhGH). CASUÍSTICA E MÉTODOS: Quatro meninas pré-púberes com baixa estatura, duas com síndrome de Turner (ST) e duas com deficiência de hormônio de crescimento (DGH). O desenvolvimento das mamas (Tanner II e III) ocorreu com idade cronológica (IC) de 5,6 e 7,7 anos e idade óssea (IO) de 5,7 a 6,9 anos, 2 a 60 meses após o inicio do tratamento com rhGH na dose de 0,1 - 0,15U/kg/d. Todas as pacientes apresentaram regressão espontânea da telarca num período de 8 a 15 meses. Três pacientes foram submetidas ao teste de estímulo com GnRH apresentando resposta pré-puberal de LH. DISCUSSÃO: O desenvolvimento de mamas após o início do tratamento com rhGH tem sido relatado em meninos, mas não em meninas pré-púberes. Concluímos que o rhGH pode induzir ao desenvolvimento transitório das mamas, também em meninas, sem a ativação do eixo hipotálamo-hipófise-gonadal, não se fazendo necessária a supressão da puberdade. (Arq Bras Endocrinol Metab 2001;45/6:547-551)

Unitermos: Desenvolvimento de mamas; Telarca; Tratamento com hormônio de crescimento.

\section{ABSTRACT}

OBJECTIVE: Description of 4 cases of breast development in prepubertal girls treated with recombinant human growth hormone (rhGH). PATIENTS AND METHODS: Four prepubertal girls with short stature: 2 with Turner's syndrome (TS) and 2 with growth hormone deficiency (GHD). Breast development (Tanner II and III) was detected from ages 5.6 to 7.7 years and bone ages from 5.7 to 6.9 years, from 2 to 60 months after starting rhGH treatment doses of $0.1-0.15 \mathrm{U} / \mathrm{kg} / \mathrm{d}$. Breast development had disappeared 8 to 15 months after have been noted in all patients. Three patients were submitted to $\mathrm{GnRH}$ test that showed a prepubertal LH response. DISCUSSION: Prepubertal breast development has been reported in boys after rhGH treatment, but not in prepubertal girls. CONCLUSION: rhGH can induce transient breast development, even in girls, without activation of the hypothalamic-pituitary-gonadal axis and these patients do not need suppression of puberty. (Arq Bras Endocrinol Metab 2001;45/6:547-551)

Keywords: Breast development; Thelarche; Growth hormone therapy.

\section{artigo original}

\author{
Luciani R.S. Carvalho \\ Maria G.F. Osorio \\ Lidia $\Upsilon$. Mimura \\ Vivian Estefan \\ Berenice B. Mendonça \\ Ivo J.P. Arnbold
}

\footnotetext{
Unidade de Endocrinologia do Desenvolvimento, Disciplina de Endocrinologia do HC-FMUSP, São Paulo, SP.
}

\begin{abstract}
A gineCOMASTIA OCORRE EM ATÉ $70 \%$ dos meninos durante a puberdade (1), mas é rara no período pré-puberal (2). Na literatura, foram relatados casos de ginecomastia em meninos pré-púberes durante o tratamento com hormônio de crescimento recombinante humano (rhGH) (3). A fisiopatologia da ginecomastia induzida por rhGH ainda é desconhecida, mas aventa-se a possibilidade de ação direta do GH sobre o tecido mamário. $\mathrm{O}$ desenvolvimento mamário em meninas pré-púberes tratadas com rhGH não foi relatado na literatura. No sexo feminino, o desenvolvi-
\end{abstract}

Recebido em 12/09/00 Revisado em 25/05/01 e 28/07/01 Aceito em 30/07/01 
mento mamário poderia ser confundido com o desenvolvimento puberal normal, indicativo de tratamento supressivo com análogos do GnRH em casos cuja estatura fosse inadequada ao início da puberdade (4).

Descrevemos a presença de desenvolvimento transitório de mamas em 4 meninas pré-púberes durante o tratamento com rhGH.

\section{CASUÍSTICA E MÉTODOS}

Na Disciplina de Endocrinologia e Metabologia do Hospital das Clínicas foram tratados com rhGH, entre os anos 1995 e 1999, 159 pacientes (83 do sexo feminino), das quais 30 com o diagnóstico de síndrome de Turner (ST). Quatro meninas pré-púberes apresentaram aparecimento de mamas bilateralmente durante o tratamento com rhGH, duas portadoras da síndrome de Turner e 2 de deficiência de GH (em uma delas associado ao hipotiroidismo hipotalâmico). O diagnóstico de deficiência de $\mathrm{GH}$ foi realizado por níveis máximos de GH após 2 testes de estímulo (clonidina $0,1 \mathrm{mg} / \mathrm{m}^{2}$ VO e insulina [ITT] $0,1 \mathrm{U} / \mathrm{kg}, \mathrm{EV}$ ) menores do que $3,2 \mathrm{ng} / \mathrm{ml}$ pelo método imunofluorométrico (5). O diagnóstico de hipotiroidismo hipotalâmico foi baseado em TSH basal elevado, hiper-resposta e manutenção prolongada (tempos 60 e 90 minutos) dos níveis de TSH em reposta ao estímu-

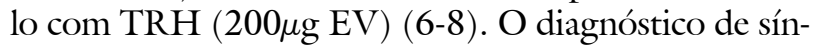
drome de Turner foi confirmado pelo cariótipo. Foram realizadas dosagens basais de LH, FSH e E2 pelo método imunofluorométrico (AutoDELFIA, Wallac, Turku, Finland). Foram considerados valores pré-púberes os basais de $\mathrm{LH}<0,6 \mathrm{UI} / \mathrm{L}$ e pico de $\mathrm{LH}$ após estímulo com GnRH <6,9UI/L (9). Três pacientes foram submetidas ao teste de estímulo com GnRH (RELISORM®), 100mg, EV. As dosagens de IGF-1 (após extração com etanol) e IGFBP-3 foram realizadas pelo método de radioimunoensaio (DSL,
Webster, TX, USA). Os valores de IGF-1 e IGFBP-3 foram expressos em escores de desvio padrão (DP) para uma população normal de mesmo sexo e idade. Os valores de TSH ultra-sensível, T4, T4 livre, T3 foram dosados pelo método imunofluorométrico por equipamento automatizado Auto-Delphia (Perkin Elmer do Brasil, Wallac, Turku, Finlândia). A idade óssea foi avaliada pelo raio-X de mão e punho de acordo com os critérios de Greulich - Pyle. O ultra-som (US) pélvico foi realizado em 2 pacientes antes do tratamento com rhGH e em 3 pacientes durante o aparecimento de mamas.

\section{RELATO DOS CASOS}

Os dados clínicos e laboratoriais estão na tabela 1 .

Caso 1 - A paciente nasceu a termo com $49 \mathrm{~cm}$ de comprimento e de peso $3060 \mathrm{~g}$, a partir dos 5 anos de idade foi observada diminuição da velocidade de crescimento. Aos 7 anos apresentava ao exame físico altura de $111,5 \mathrm{~cm}(-1,42 \mathrm{DP})$ e estigmas típicos da síndrome de Turner. O cariótipo foi 45, X/46 X, i (Xq), caracterizando a síndrome de Turner. O US de rins era normal e o US pélvico antes do tratamento com rhGH evidenciou útero infantil e ovários não visualizados. A função tireoideana estava normal $[\mathrm{T} 4 \mathrm{~L}=1, \mathrm{lng} / \mathrm{dL}$ (normal $=0,6-1,54 \mathrm{ng} / \mathrm{dL}$ ); $\mathrm{TSH}=2 \mu \mathrm{U} / \mathrm{mL}$ (nor$\mathrm{mal}=0,5-4,2 \mu \mathrm{U} / \mathrm{mL})]$. O tratamento com rhGH foi iniciado na dose de $0,15 \mathrm{U} / \mathrm{Kg} / \mathrm{d}, \mathrm{sc}$, à noite, 7 dias por semana, e aos 7,7 anos (7 meses após início do rhGH), foi notado aparecimento de mamas bilateralmente (Tanner II), que regrediram espontaneamente 3 meses após o seu aparecimento, na vigência do uso de rhGH. A idade óssea nesse período era de 5,8 anos, e os exames laboratoriais basais evidenciavam $\mathrm{LH}<0,6 \mathrm{U} / \mathrm{L}$, FSH $2,8 \mathrm{U} / \mathrm{L}$ e E2 < $13 \mathrm{pg} / \mathrm{ml}$. Os níveis de IGF-1 e IGFBP-3, antes do tratamento com rhGH, eram de

Tabela 1. Dados clínicos e laboratoriais de 4 pacientes pré púberes que apresentaram telarca durante tratamento com hormônio de crescimento.

\begin{tabular}{|c|c|c|c|c|c|c|c|c|c|c|c|}
\hline \multirow[t]{2}{*}{ Caso } & \multirow[t]{2}{*}{ Diagnóstico } & \multicolumn{2}{|c|}{$\begin{array}{l}\text { Início da telarca } \\
\text { (anos) }\end{array}$} & \multirow[t]{2}{*}{$\begin{array}{c}\text { Dose de GH } \\
\mathrm{U} / \mathrm{Kg} / \mathrm{d}\end{array}$} & \multicolumn{2}{|c|}{$\begin{array}{l}\text { LH } \\
(\mathrm{U} / \mathrm{L})\end{array}$} & \multicolumn{2}{|c|}{$\begin{array}{l}\text { FSH } \\
(\mathrm{U} / \mathrm{L})\end{array}$} & \multirow[t]{2}{*}{$\begin{array}{c}\text { E2 } \\
(p g / m l)\end{array}$} & \multirow[t]{2}{*}{$\begin{array}{l}\text { IGF-1* } \\
\text { SDS }\end{array}$} & \multirow[t]{2}{*}{$\begin{array}{l}\text { IGFBP3* } \\
\text { SDS }\end{array}$} \\
\hline & & IC & 10 & & B & $P$ & B & $P$ & & & \\
\hline 1 & Síndrome Turner & 7,7 & 5,8 & 0,15 & 0,6 & --- & 28 & --- & $<13$ & $0 a+1$ & 0 \\
\hline 2 & Síndrome Turner & 7,2 & 6,9 & 0,10 & $<0,6$ & 5,3 & 6,3 & 45 & $<13$ & $0 e-1$ & 0 e - 1 \\
\hline 3 & Deficiência GH & 5,6 & 5,7 & 0,10 & $<0,6$ & 3,1 & 1,1 & 10 & $<13$ & $+1 a+2$ & $+1 a+2$ \\
\hline 4 & Deficiência GH & 7,9 & 6,0 & 0,10 & $<0,6$ & $<0,6$ & $<1,0$ & 3,6 & $<13$ & 0 e -1 & 0 e -1 \\
\hline
\end{tabular}

IC: Idade cronológica; IO: Idade óssea; B: Basal; P: Pico após teste de GnRH

* Dosagens hormonais durante o tratamento com $\mathrm{GH}$ 
$478 \mathrm{ng} / \mathrm{ml}($ entre +1 e $+2 \mathrm{DP}$ ) e $3,2 \mathrm{mg} / \mathrm{l}$ (entre - 1 e 0 $\mathrm{DP})$, respectivamente; durante o tratamento com $\mathrm{rhGH}, 227 \mathrm{ng} / \mathrm{ml}$ (entre 0 e $+1 \mathrm{DP})$ e $3,5 \mathrm{mg} / \mathrm{l}$ (0 DP), respectivamente. A paciente encontra-se atualmente com $\mathrm{IC}=9,1$ anos e $\mathrm{IO}=8,8$ anos, não se encontra em reposição hormonal com estrógenos e ainda não apresentou desenvolvimento dos caracteres sexuais secundários.

Caso 2 - Paciente nascida a termo com comprimento de $46 \mathrm{~cm}$ e peso $2600 \mathrm{~g}$, sem intercorrências pré, peri e pós-natais maternas ou fetais. Apresentou otites médias agudas de repetição. Procurou ambulatório de endocrinologia aos 7 anos com quadro de baixa estatura. Apresentava ao exame físico altura de $105,5 \mathrm{~cm} \mathrm{(-}$ $2,61 \mathrm{DP})$, epicanto, palato ogival, implantação baixa das orelhas, tórax em escudo, cúbito valgo, quarto metacarpo curto e nevus pigmentado em face, dorso, abdome. O cariótipo foi $45, \mathrm{X}, 46 \mathrm{X},+\mathrm{r}$ sendo diagnosticado síndrome de Turner. O exame de US de rins foi normal e o US pélvico antes do tratamento com rhGH evidenciou útero infantil e ovários não visualizados. A função tireoideana estava normal $\mathrm{TSH}=3, \mathrm{l} \mu \mathrm{U} / \mathrm{mL}$; $\mathrm{T} 4 \mathrm{~L}=1,2 \mathrm{ng} / \mathrm{dL}$. Aos 7,1 anos foi iniciado tratamento com GH $0,1 \mathrm{U} / \mathrm{kg} / \mathrm{d}$, sc, à noite, 7 dias por semana. Dois meses após o início do tratamento com rhGH, foi observado aparecimento de mamas bilateralmente (Tanner III), seguida de regressão espontânea 8 meses após na vigência do tratamento com rhGH. Na época do aparecimento das mamas a idade óssea era 6,9 anos, o nível de $\mathrm{E} 2<\mathrm{l} 3 \mathrm{pg} / \mathrm{ml}$; o teste de estímulo com GnRH evidenciou resposta pré-puberal (tabela 1). Os níveis de IGF-1 e IGFBP-3 antes do tratamento com rhGH eram de $41 \mathrm{ng} / \mathrm{ml}$ (entre -3 e - 2 DP) e $1,55 \mathrm{mg} / \mathrm{l}$ (entre -3 e -2 DP), respectivamente, durante tratamento com rhGH, $147 \mathrm{ng} / \mathrm{ml}$ (entre 0 e $-1 \mathrm{DP}$ ) e $3,2 \mathrm{mg} / \mathrm{l}$ (entre 0 e $-1 \mathrm{DP})$, respectivamente. O US pélvico durante o desenvolvimento de mamas evidenciou útero de $1,25 \mathrm{ml}$, ovário $\mathrm{D}$ de $0,5 \mathrm{ml}$ e ovário $\mathrm{E}$ não visualizado. A paciente apresenta atualmente $\mathrm{IC}=9,5$ anos e $\mathrm{IO}=8,8$ anos não houve recidiva da telarca, encontra-se ainda pré-púbere sem reposição de estrógenos.

Caso 3 - Paciente nasceu com $43 \mathrm{~cm}$ e peso de 2620g. Aos 2,4 anos foi notada diminuição da velocidade de crescimento. Ao exame físico apresentava altura de $74,4 \mathrm{~cm}$ (-5 DP), fronte olímpica e clinodactilia. Os teste de estímulo com clonidina e ITT não promoveram liberação de $\mathrm{GH}$ (pico máximo de $0,9 \mathrm{ng} / \mathrm{mL}$ ) indicando o diagnóstico de deficiência de GH. A função tireoideana estava normal $\mathrm{TSH}=0,7 \mu \mathrm{U} / \mathrm{L}$ e $\mathrm{T} 4 \mathrm{~L}=$
$0,8 \mathrm{ng} / \mathrm{dl}$. Aos 2,7 anos, com altura de 76,4cm (-5 DP), foi iniciado tratamento com rhGH na dose de $0,1 \mathrm{U} / \mathrm{kg} / \mathrm{dia}, \mathrm{SC}$, à noite, 7 vezes por semana. A paciente apresentou desenvolvimento de mamas bilateralmente (Tanner II) aos 5,6 anos, 2 anos e 10 meses após o início da administração de rhGH que evoluiu para Tanner III em 2 meses. Aos 6,7 anos ( 1 ano e 3 meses após o início da telarca) houve regressão espontânea sem suspensão do tratamento com rhGH, sendo que 4 meses após a regressão foi notado recidiva de mamas bilateralmente (Tanner II a direita e Tanner III a esquerda), com progressão para Tanner IV. A idade óssea durante o aparecimento de mamas era de 5,7 anos quando a idade cronológica era 5,6 anos. Os níveis basais de LH, FSH e E2 eram pré-púberes e o teste de estímulo com $\mathrm{GnRH}$ evidenciou padrão pré-puberal tanto aos 5,6 anos na vigência de mamas (tabela 1 ) como aos 7,9 anos durante a recidiva da telarca $(\mathrm{LH}$ basal <0,6, pico 3,6U/L, FSH basal 1,0U/L, pico $8,5 \mathrm{U} / \mathrm{L})$. O US pélvico evidenciou durante aparecimento das mamas útero de $2,7 \mathrm{ml}$, ovário $\mathrm{D}$ de $1,9 \mathrm{ml}$ com microcistos, o maior medindo $6,0 \mathrm{~mm}$, e o ovário $\mathrm{E}$ de $1,3 \mathrm{ml}$; após a regressão da telarca, útero de $2,6 \mathrm{ml}$, ovário $\mathrm{D}$ de $1,8 \mathrm{ml}$ e ovário $\mathrm{E}$ de $1,1 \mathrm{ml}$, ambos com microcistos no interior de até $6 \mathrm{~mm}$; durante recidiva útero de $1,8 \mathrm{ml}$, ovário D de $1,6 \mathrm{ml}$ e ovário $\mathrm{E}$ de $1,6 \mathrm{ml}$; e $\mathrm{l}$ ano após a recidiva evidenciou útero de $2, \mathrm{lml}$, ovário D de $2,4 \mathrm{ml}$, ovário $\mathrm{E}$ de $1,4 \mathrm{ml}$, sem cistos. Os níveis de IGF-1 e de IGFBP-3 foram respectivamente, antes do tratamento com rhGH, 16ng/ml (entre -2 e -3 DP) e $1,9 \mathrm{mg} / \mathrm{l}$ (-l DP); e durante tratamento com rhGH de $239 \mathrm{ng} / \mathrm{ml}$ (entre +1 e $+2 \mathrm{DP}$ ) e $4,6 \mathrm{mg} / \mathrm{l}$ (entre +1 e $+2 \mathrm{DP})$, mantendo-se dentro dos valores normais durante todo o tratamento com rhGH.

Caso 4 - Paciente nasceu a termo com $47 \mathrm{~cm} \mathrm{e}$ peso $3620 \mathrm{~g}$. Procurou ambulatório de endocrinologia aos 3,1 anos com queixa de baixa estatura desde os 2 anos de idade. Ao exame físico apresentava altura de $7 \mathrm{lcm}(-5,0 \mathrm{DP})$, fronte olímpica e nariz em sela. $\mathrm{Na}$ investigação diagnóstica, não apresentou resposta normal do $\mathrm{GH}$ aos testes de clonidina ( $\mathrm{GH}$ basal $0,5 \mathrm{e}$ pico $3,1 \mathrm{ng} / \mathrm{ml}$ ) e ITT ( $\mathrm{GH}$ basal 0,4 e pico $0,8 \mathrm{ng} / \mathrm{ml}$ ). O T4 livre era 1,0ng/dl e houve hiper-resposta do TSH ao TRH com padrão de resposta prolongada, compatível com deficiência hipotalâmica (TSH basal 5,27 e pico $49 \mu \mathrm{U} / 1$ aos 90 minutos), indicando diagnóstico de deficiência de GH e de TRH. Aos 4,1 anos de idade, com altura de $82 \mathrm{~cm}(-4,9 \mathrm{DP})$, foi iniciado tratamento com rhGH $0,1 \mathrm{U} / \mathrm{kg} / \mathrm{dia}, \mathrm{SC}$, à noite, 7 vezes por semana, e tiroxina $75 \mu \mathrm{g} / \mathrm{dia}$, apresentando níveis eutireóideos após 5 meses do início da terapêu- 
tica com T4 livre $=1,5 \mathrm{ng} / \mathrm{dL}$ e $\mathrm{TSH}=0,04 \mu \mathrm{U} / \mathrm{mL}$. Apresentou mamas (Tanner II) aos 7,9 anos (5 anos após o início de tratamento com rhGH), apresentando regressão espontânea aos 8,1 anos (4 meses após o início das mamas) sem interrupção do tratamento com rhGH. A avaliação laboratorial em vigência de mamas mostrava níveis basais de LH, FSH e E2 prépúberes e o teste de estímulo com GnRH evidenciou padrão pré-puberal (tabela 1). O US pélvico durante o aparecimento de mamas evidenciou útero de $2,3 \mathrm{ml}$, ovário $\mathrm{D}$ de $1,4 \mathrm{ml}$ e ovário $\mathrm{E}$ de $2,9 \mathrm{ml}$, com pequenos folículos periféricos. O US pélvico após a regressão das mamas evidenciou útero de $1,5 \mathrm{ml}$, ovário $\mathrm{D}$ de $1,2 \mathrm{ml}$ e ovário E de $1,9 \mathrm{ml}$. Os níveis de IGF-1 e IGFBP-3 durante o aparecimento de mamas foram, respectivamente, de $14 \mathrm{lng} / \mathrm{ml}$ (entre 0 e -1 DP) e $3,4 \mathrm{mg} / \mathrm{l}$ (entre 0 e $-1 \mathrm{DP}$ ) e após a regressão $34 \mathrm{lng} / \mathrm{ml}$ (entre 0 e +1 DP) e 4,2mg/l (entre 0 e +1 DP).

As 4 pacientes avaliadas negavam doenças associadas ou uso de outras drogas concomitantes e a presença de mamas ao nascimento ou antes do início de uso de rhGH.

\section{DISCUSSÃO}

O desenvolvimento de mamas durante tratamento com rhGH, com involução espontânea, foi observado em 21 meninos pré-púberes 0,5 a 96 meses após o início de tratamento (3). Não há relatos na literatura de desenvolvimento de mamas em meninas pré-púberes durante tratamento com rhGH. Observamos aparecimento de mamas 2 a 60 meses após início de tratamento com rhGH em 4 meninas. Apesar da continuidade do tratamento com rhGH, houve regressão em todas as pacientes entre 3 meses e 15 meses após o início do aparecimento das mamas, sendo que uma paciente apresentou recidiva 4 meses após a regressão. $\mathrm{O}$ aparecimento da mama não foi acompanhado de alterações laboratoriais ou avanço da idade óssea compatíveis com puberdade precoce dependente de gonadotrofinas e os níveis de IGF-1 e IGFBP-3 durante $\mathrm{o}$ aparecimento das mamas não foram maiores que $+2 \mathrm{DP}$.

O desenvolvimento precoce de mamas, a telarca prematura, ocorre na infância numa incidência de 21,2 por 100.000 pacientes/ano, geralmente entre os 2 e 4 anos; o aumento da mama regride após meses, mas ocasionalmente persiste por anos ou dura até a puberdade normal (10). Em nossas pacientes o aparecimento das mamas não ocorreu entre a época esperada para telarca prematura e foi associado ao uso de
rhGH. O mecanismo responsável pelo aparecimento de ginecomastia ou telarca durante o tratamento com rhGH não está estabelecido, porém algumas considerações devem ser feitas sobre o papel do GH na reprodução feminina. Receptores de GH foram identificados em linhagens de células de mama humanas (11). O rhGH poderia estimular o crescimento das mamas diretamente através dos receptores de $\mathrm{GH}$, de outros receptores lactogênicos (12), ou indiretamente através da produção de fatores de crescimento (13). O efeito estimulatório do GH na mamogênese e lactogênese foi estabelecido por vários estudos in vivo e in vitro (14). O envolvimento do IGF-1 local e hepático nessas ações parece ser espécie dependente. A glândula mamária é também local de síntese de $\mathrm{GH}$ e o $\mathrm{GH}$ mamário provavelmente age localmente por um mecanismo autócrino/parácrino (14).

O ovário possui receptores de GH e a ação desse hormônio potencializa a esteroidogênese e a gametogênese (15). O GH estimula a síntese de progesterona e estrogênio. A ação do GH na esteroidogênese está associada ao aumento da atividade de muitas enzimas e pode ser parcialmente responsável pelo efeito facilitatório do GH sobre a foliculogênese e gametogênese (14); o GH estimula a esteroidogênese na espécie humana por ação direta independente da IGF-1 (16). As pacientes neste estudo apresentavam níveis de estradiol inferiores a $13 \mathrm{pg} / \mathrm{ml}$, afastando uma secreção estrogênica importante e contínua. No entanto, um discreto e moderado aumento dos ovários, detectados respectivamente ao exame de US das pacientes 3 e 4 , sugere uma participação ovariana nestas pacientes.

Mazzanti e cols. relataram que $50 \%$ das meninas portadoras de síndrome de Turner com cariótipo mosaico apresentaram telarca espontânea e $38 \%$ tiveram menarca espontânea (17). Estas pacientes apresentavam níveis normais de gonadotrofinas durante a puberdade e ovários bilaterais estavam presentes ao exame de ultra-som (17). Ao contrário, em nossa casuística uma das pacientes com síndrome de Turner apresentou hiper-resposta de FSH após estímulo com GnRH, e a outra paciente com a síndrome apresentava níveis basais já elevados de FSH, indicativos da presença de disgenesia gonadal.

Cohn e cols. descreveram ginecomastia em homens adultos idosos após administração de rhGH, que ocorreu quando houve aumento nas concentrações de IGF-1 (18). Nossos dados não demonstraram relação positiva entre o aparecimento da mama e as dosagens de IGF-1 e IGFBP-3 que oscilaram entre -3 $\mathrm{DP}$ e $+2 \mathrm{DP}$, antes e após desenvolvimento da telarca. 
Concluímos que o desenvolvimento de mamas em meninas pré-púberes induzido pelo tratamento com rhGH ocorreu em $5 \%$ da nossa casuística e foi caracterizado por curso transitório, sem necessidade de terapêutica. Em meninas com déficit de GH, o desenvolvimento precoce de mamas poderia indicar o início da puberdade e a necessidade de bloqueio puberal com análogos do GnRH, visando preservação da altura final. Considerando a possibilidade da telarca $\mathrm{GH}-$ induzida, sugerimos que a avaliação do eixo hipotálamo-hipófise-gonadal seja realizada antes de iniciar tratamento com análogo de GnRH.

\section{REFERÊNCIAS}

1. Nydick M, Bustos J, Dale J, Rawson RW. Gynecomastia in adolescent boys. JAMA 1961;178:449-54.

2. Haibach H, Rosebholtz MJ. Prepubertal gynecomastia with lobules and acini: a case report and review of the literature. Am J Clin Pathol 1983;80:252-5.

3. Malozowski S, Stadel V. Prepubertal gynecomastia during growth hormone therapy. J Pediatr 1995;126:659-61.

4. Merica V, Eggers M, Avila A, Culter GB Jr, Cassorla F. Near final height in pubertal growth hormone $(\mathrm{GH})$ - deficient patients treated with $\mathrm{GH}$ alone or in combination with luteinizing hormone-releasing hormone analog: Results of a prospective randomized trial. J Clin Endocrinol Metab 2000;85:569-73.

5. Silva EGP, Batista MC, Arnhold IJP, Mazi CR, Goto SY, Nicolau W, et al. Horm Res 1997;48(suppl 2):88 (abstract 434).

6. Martino E, Bartalena L, Faglia G, Pinchera A. Central hypothyroidism. In: Braverman LE, Utiger RD, eds. Werner and Ingbar's The Thyroid, 7th Ed. Philadelphia: LippincottRaven 1996;779-91.

7. Patel YC, Burger HG. Serum thyrotropin in pituitary and/or hypothalamic hypothyroidism. J Clin Endocrinol Metab 1973;37:190-6.

8. Faglia G, Beck-Peccoz P, Ferrari C, et al. Plasma thyrotropin response to thyrotropin-releasing hormone in patients with pituitary and hypothalamic disorders. J Clin Endocrinol Metab 1973;37:595-601.

9. Brito VN, Batista MC, Latronico AC, Kohek MB, Arnhold IJ, Mendonça BB, et al. Diagnostic value of fluorometric assay in the evaluation of precocious puberty. J Clin Endocrinol Metab 1999;84:3539-44.
10. Styne DM, Grumbach MM. Variations of pubertal development. In: Wilson, Foster, Kronenberg, Larsen, eds. Williams Textbook of Endocrinology, 9th Ed. WB Saunders Co.:Philadelphia 1998, pg 1599.

11. Murphy LJ, Murphy LC, Stead B, Sutherland RL, Lazarus L. Modulation of lactrogenic receptors by progestins in cultured human breast cancer cells. J Clin Endocrinol Metab 1986;62:280-7.

12. Fradkin JE, Eastman RC, Lesniak MA, Roth J. Specificity spillover at the hormone receptor: exploring its role in human disease. N Engl J Med 1989;320:640-5.

13. Rosen N, Yee D, Lippman ME, Paik S, Cullen KJ. Insulin like growth factors in human breast cancer. Breast Cancer Res Treat 1991;18:S55-62.

14. Hull KL, Harvey S. Growth Hormone: roles in female reproduction. J Endocrinol 2001;168,1-23.

15. Sharara FI, Giudice LC. Role of growth hormone in ovarian physiology and onset of puberty. J Soc Gynecol Investig 1997;4:2-7.

16. Ovesen P, Ingerslev J, Orskov H, Ledet T. Effect of growth hormone and steroidogenesis, insulin-like growth factor-I (IGF-1) and IGF-binding protein-I production and DNA synthesis in cultured human luteinized granulose cells. J Endocrinol 1994;140:313-9.

17. Mazzanti L, Cacciari E, Bergamaschi R, Tassinari D, Magnani $C$, Perri $A$, et al. Pelvic ultrasonography in patients with Turner syndrome: age-related findings in different karyotypes. J Pediatr 1997;131:135-40.

18. Cohn I, Feller AG, Draper MW, Rudman IW, Rudman D. Carpal Tunnel syndrome and gynecomastia during growth hormone treatment of elderly men with low circulating IGF1 concentrations. Clin Endocrinol 1993;39: 417-25.

\section{Endereço para correspondência:}

Ivo J.P. Arnhold

Divisão de Endocrinologia - la C.M. Hospital das Clínicas

Caixa postal 3671

01060-970 São Paulo, SP

FAX: (011) 3083-0626 